\title{
Procedurally fair income taxation schemes
}

Citation for published version (APA):

Herings, P. J. J., \& Predtetchinski, A. (2011). Procedurally fair income taxation schemes. METEOR, Maastricht University School of Business and Economics. METEOR Research Memorandum No. 035 https://doi.org/10.26481/umamet.2011035

Document status and date:

Published: 01/01/2011

DOI:

10.26481/umamet.2011035

Document Version:

Publisher's PDF, also known as Version of record

\section{Please check the document version of this publication:}

- A submitted manuscript is the version of the article upon submission and before peer-review. There can be important differences between the submitted version and the official published version of record.

People interested in the research are advised to contact the author for the final version of the publication, or visit the DOI to the publisher's website.

- The final author version and the galley proof are versions of the publication after peer review.

- The final published version features the final layout of the paper including the volume, issue and page numbers.

Link to publication

\footnotetext{
General rights rights.

- You may freely distribute the URL identifying the publication in the public portal. please follow below link for the End User Agreement:

www.umlib.nl/taverne-license

Take down policy

If you believe that this document breaches copyright please contact us at:

repository@maastrichtuniversity.nl

providing details and we will investigate your claim.
}

Copyright and moral rights for the publications made accessible in the public portal are retained by the authors and/or other copyright owners and it is a condition of accessing publications that users recognise and abide by the legal requirements associated with these

- Users may download and print one copy of any publication from the public portal for the purpose of private study or research.

- You may not further distribute the material or use it for any profit-making activity or commercial gain

If the publication is distributed under the terms of Article $25 \mathrm{fa}$ of the Dutch Copyright Act, indicated by the "Taverne" license above, 


\section{Maastricht University}

P. Jean-Jacques Herings,

A. Predtetchinski

Procedurally Fair Income Taxation Schemes

$\mathrm{RM} / 11 / 035$

\section{METEOR}

Maastricht University School of Business and Economics

Maastricht Research School of Economics

of Technology and Organization

PO. Box 616

NL-6200 MD Maastricht

The Netherlands 


\title{
Procedurally Fair Income Taxation Schemes
}

\author{
P. Jean-Jacques Herings* $\quad$ A. Predtetchinski ${ }^{\dagger}$
}

June 23, 2011

\begin{abstract}
We study the implications of procedural fairness on income taxation. All taxation schemes are allowed for, up to the constraints implied by incentive compatibility and budget balance. We formulate procedural fairness as a particular non-cooperative bargaining game and examine the stationary subgame perfect equilibria of the game. The equilibrium outcome is called tax equilibrium and is shown to be unique. Procedurally fair taxation schemes are defined as the taxation schemes that result in the limit of tax equilibria when the probability that negotiations break down converges to zero. The procedurally fair taxation scheme is shown to be unique. It is shown to correspond to the taxation scheme resulting from the Nash bargaining solution and leads to full redistribution of income.
\end{abstract}

KEYworDS: Procedural fairness, income taxation, non-linear taxation schemes, bargaining.

JEL CODES: C78, D63, H24.

*P.J.J. Herings, Department of Economics, Maastricht University. This author would like to thank the Netherlands Organization for Scientific Research (NWO) for financial support.

$\dagger^{\dagger}$ A. Predtetchinski, Department of Economics, Maastricht University. This author would like to thank the Netherlands Organization for Scientific Research (NWO) for financial support. 


\section{Introduction}

Modern societies redistribute large amounts of income by means of income taxation in an attempt to improve upon the fairness of the income distribution. In the theory of income taxation it is standard practise to view the problem of choosing a taxation scheme as an optimization problem by a social planner as is for instance the case in the landmark contribution by Mirrlees (1971) and many of his followers. A problematic part of this approach is that there is no reason to favor one social welfare function over another. In general, few attempts have been made to justify the particular social welfare function chosen, a notable exception being Fleurbaey and Maniquet (2006), who base the social welfare function on the Pigou-Dalton transfer principle.

Cooperative game theory has taken a different approach. The problem of fair income taxation is frequently studied, though it is usually formulated as a bankruptcy problem. The typical approach there is to specify axioms that a taxation scheme should satisfy and to show that the axioms pin down a unique solution. Many taxation schemes can be justified by the axiomatic approach. Well-known rules that have been axiomatized are the proportional, adjusted proportional, constrained equal awards, constrained equal losses, Piniles', egalitarian, constrained egalitarian, and the random arrival rule. For a survey of this approach we refer the reader to Thomson (2003). Incentive issues, central to the work of Mirrlees (1971), are typically ignored in the cooperative approach.

In this paper we analyze procedural fairness rather than fairness, and use non-cooperative game theory to analyze the taxation schemes that result from using a fair procedure to decide upon income taxation. Procedures are considered fair if they create equal chances for persons involved in the procedure. It is equal opportunities that matters rather than an equal allocation that results. This approach allows use to analyze fair choice without the ad hoc imposition of a social welfare function. At the same time we can easily deal with incentive issues, as our approach benefits from the full flexibility offered by non-cooperative game theoretic modeling.

The fair procedure that we consider is called the unanimous approval procedure. In the unanimous approval procedure, all citizens in society have an equal chance of making a proposal. A proposal is only carried out if it is approved of by all citizens. If one of the citizens rejects, there is some probability that the procedure breaks down. With the remaining probability, the continuation probability, a new proposer is selected at random from the pool of all citizens, and so on, and so forth.

We propose the unanimous approval procedure as a normative concept. Since people tend to object strongly to procedures that are not deemed fair (Bolton, Brandts, and Ockenfels, 2005), it may have value as a positive concept too. The unanimous approval procedure resembles an ideal direct democracy where every citizen has equal rights in 
making and rejecting proposals. Although ideal direct democracies do not exist, many democratic procedures have the intention to approximate such an ideal situation.

The unanimous approval procedure is an extension of the alternating offers procedure introduced in Rubinstein (1982) to the situation with an arbitrary number of citizens. Contrary to Rubinstein (1982), citizens do not alternate or rotate in making offers, but are selected randomly in each bargaining round, as in Binmore (1987). The unanimous approval procedure is also a special case of the bargaining models of collective choice as considered in Banks and Duggan (2000), where the recognition probabilities are restricted to be uniform and approval by a coalition within a set of decisive coalitions is replaced by unanimous approval. Banks and Duggan (2000) study such models as positive rather than normative concepts.

The study of procedural fairness in income taxation was initiated in Herings and Predtetchinski (2011b). In that paper it was assumed that taxation schemes have to be linear and the problem studied was therefore the determination of the procedurally fair tax rate. The paper shows that the procedurally fair tax rate is unique. It also provides a general characterization of the procedurally fair tax rate. Next this characterization is used to demonstrate that in societies populated by citizens with constant relative risk aversion, as well as in sufficiently heterogeneous societies, the procedurally fair tax rate is equal to the probability mass of below average income citizens.

In this paper we de not make any assumptions on taxation schemes, beyond the ones imposed by incentive compatibility. Societies are populated by citizens with different productivity levels. Citizens do not derive disutility from labor. Incentive compatibility then requires that after-tax income be increasing in pre-tax income. Taxation is used for redistributive purposes only, so the second constraint on feasible taxation schemes comes from a balanced budget requirement. Citizens are free to propose any taxation scheme satisfying these two constraints. In particular, they may propose a complete redistribution of income, the egalitarian solution, they may propose laissez-faire, and they may even propose to tax the poor and subsidize the rich. Since taxation schemes are general up to the two constraints, we can also address whether procedurally fair taxation leads to progressive income taxation, an issue taken up in Roemer (1999) and a large subsequent literature.

A tax equilibrium is a tuple of proposals, one for each citizen, with the property that all proposals are accepted by all citizens. Moreover, the proposal of a citizen is the one giving him the highest income among all proposals that are accepted by all citizens. We argue that tax equilibria are in a one-to-one correspondence with subgame perfect equilibria in stationary strategies of the extensive form game implied by the unanimous approval procedure.

In the presence of incentive compatibility constraints, a complete redistribution of in- 
come is the most extreme egalitarian proposal that is feasible. The most extreme proposal in the other direction is to fully tax the income of all citizens except the most productive one, and transfer it to the citizen with highest productivity. Although this proposal is feasible, it will not be proposed by any citizen, not even the one with the highest productivity, since it is surely rejected by the other citizens. A proposal involving complete redistribution will also not be proposed by the citizen with the highest productivity. Incentive compatibility implies that this citizen receives at least the average income in all proposals, and strictly more if he is proposing himself.

Consider a tax equilibrium proposal by a particular citizen. All citizens with lower productivity are taxed up to the reservation income derived from their tax equilibrium utility. To avoid rejection by more productive citizens, also those have to be promised at least their reservation income. Incentive compatibility of the taxation scheme may force the citizen to make a proposal strictly above their reservation income. In general, the tax equilibrium proposal is such that all citizens with higher productivity up to some productivity level are proposed the same after-tax income as the proposer, and citizens with even higher productivity their reservation income.

In general multi-lateral bargaining models with unanimous agreement, little is known about conditions under which equilibria are unique. The most general results are derived by Merlo and Wilson (1995). However, the incentive compatibility constraints on taxation schemes make that their assumptions on the set of feasible alternatives are violated. Nevertheless, we can show that tax equilibria are unique.

Consider a sequence of continuation probabilities converging to one and consider the corresponding sequence of tax equilibria. It follows from a standard argument that all citizens make the same proposal in the limit. Taxation schemes are said to be procedurally fair if they correspond to such a limit proposal. We are interested in the uniqueness and the characterization of procedurally fair taxation schemes.

The literature on multi-lateral bargaining with unanimous agreement has shown convergence of bargaining equilibrium proposals to the Nash bargaining solution (Hart and Mas-Colell (1996), Laruelle and Valenciano (2007), Miyakawa (2008), Kultti and Vartiainen (2010), and Britz, Herings and Predtetchinski (2010)). Unfortunately, all these papers need differentiability assumptions with respect to the set of feasible alternatives, an assumption that is clearly violated for the set of feasible taxation schemes. Moreover, Kultti and Vartiainen (2010) and Herings and Predtetchinski (2011a) present examples where in the absence of differentiability assumptions, sequences of bargaining equilibria may have a limit different from the Nash bargaining solution and may have multiple limits.

For the cases with two or three citizens it is possible to calculate tax equilibria explicitly and verify uniqueness of the procedurally fair taxation scheme. For these cases, it 
corresponds to the egalitarian outcome, although for the three-citizen case this outcome is not proposed by any citizen for sufficiently high values of the continuation probability, but is only reached in the limit. The egalitarian outcome, or equivalently, complete redistribution, is also the outcome predicted by an application of the Nash bargaining solution to our tax problem. We demonstrate that also in the $n$-player case, the procedurally fair taxation scheme is unique and leads to the egalitarian outcome.

Our results demonstrate a striking difference between the case where taxation schemes are required to be linear as studied in Herings and Predtetchinski (2011b) versus the case where the taxation scheme is fully flexible, up to incentive compatibility constraints. In the former case, a procedurally fair tax rate strictly less than one results and, under some additional conditions, the procedurally fair tax rate is equal to the probability mass of below average income citizens. Full flexibility of taxation schemes leads to a procedurally fair taxation scheme corresponding to full redistribution. Below average income citizens can apparently exploit the flexibility of taxation schemes to increase the amount of redistribution in society.

This paper is organized as follows. Section 2 introduces the unanimous approval procedure and Section 3 the notion of tax equilibrium. Section 4 characterizes optimal proposals of citizens and Section 5 demonstrates the uniqueness of tax equilibria. Section 6 computes the tax equilibrium explicitly for two simple examples and shows that it converges to complete redistribution in the limit when the continuation probability converges to one. Procedurally fair taxation schemes are introduced in Section 7 and they are shown to be unique and equal to the taxation scheme corresponding to the Nash bargaining solution in Section 8. Section 9 concludes.

\section{The Unanimous Approval Procedure}

We study procedurally fair taxation in a society with a finite population of citizens $N=$ $\{1, \ldots, n\}$. A citizen $i \in N$ has a productivity level of $w_{i} \geq 0$, can work up to one unit of time, and derives no disutility from labor. Citizen $i$ therefore has a pre-tax income of $w_{i}$. We assume that $w_{i}<w_{i^{\prime}}$ if $i<i^{\prime}$. The after-tax income of citizen $i$ is denoted $x_{i}$. We write $w=\left(w_{1}, \ldots, w_{n}\right)$ and $x=\left(x_{1}, \ldots, x_{n}\right)$.

For a group of citizens $G \subset N$ and a vector $y \in \mathbb{R}^{n}$, we use the notation $y(G)=\sum_{i \in G} y_{i}$. A group of citizens $G$ of the form $\{i, i+1, \ldots, j-1, j\}$ for some $i, j \in N$, is denoted $[i, j]$.

Without loss of generality we normalize aggregate pre-tax income $w(N)$ to be equal to 1. It follows that $w_{i}$ corresponds to the share of aggregate pre-tax income that is generated by citizen $i$.

We are interested in the case where taxation schemes are general up to two constraints. 
The first constraint comes from incentive compatibility considerations. After-tax income should be increasing in pre-tax income, so $x_{i} \leq x_{i^{\prime}}$ if $i \leq i^{\prime}$. The second constraint is a balanced budget requirement. We assume that taxation is used for redistribution purposes only, so aggregate after-tax income $x(N)$ should be equal to aggregate pre-tax income $w(N)$.

The set $X$ of feasible after-tax incomes is therefore equal to

$$
X=\left\{x \in \mathbb{R}_{+}^{n} \mid x_{i} \leq x_{i^{\prime}} \text { if } i \leq i^{\prime}, x(N)=1\right\} .
$$

Two possible taxation schemes are complete redistribution and laissez-faire. Complete redistribution amounts to $x_{i}=1 / n$ for all citizens $i$. In the case of laissez-faire it holds that $x_{i}=w_{i}$ for all citizens $i$. Complete redistribution is the most egalitarian feasible taxation scheme. The least egalitarian feasible taxation scheme specifies $x_{i}=0$ for $i \in[1, n-1]$ and $x_{n}=1$, and redistributes all income to the highest-income citizen.

Procedural fairness embodies equal opportunities for all citizens involved in the procedure. When applied to income taxation, we model procedural fairness as a process in which all citizens have an equal opportunity to make a proposal, and where a proposal has to be approved of by all citizens in order to be implemented. These principles lead to the unanimous approval procedure as introduced in Herings and Predtetchinski (2011b), where attention is restricted to the case of linear taxation schemes.

The unanimous approval procedure is defined as follows. In each bargaining round $r$, each citizen has an equal chance to be selected as the proposer. The selected citizen, say citizen $i$, makes a proposal $p^{i} \in X$. After observing $p^{i}$, citizens sequentially decide whether to accept or to reject the proposal in a fixed a priorily chosen order. If all citizens accept, then after-tax incomes are given by $p^{i}$, leading to utility $p_{j}^{i}$ for citizen $j \in N$. As soon as some citizen rejects, the procedure breaks down with probability $1-\delta>0$ and continues with probability $\delta \geq 0$ in bargaining round $r+1$ with the selection of a randomly selected citizen as the proposer. If no agreement is ever reached, all citizens receive utility 0.

We analyze subgame perfect equilibria in stationary strategies (SSPE) of the game induced by the unanimous approval procedure. A stationary strategy of citizen $i, \sigma^{i}=$ $\left(p^{i}, A^{i}\right)$, consists of a proposal $p^{i} \in X$ and an acceptance set $A^{i} \subset X$. The acceptance set consists of those proposals that are accepted by a citizen. This specification results in a stationary strategy because $p^{i}$ and $A^{i}$ are time and history independent. We write $p=\left(p^{1}, \ldots, p^{n}\right)$ and $A=\left(A^{1}, \ldots, A^{n}\right)$. The social acceptance set consists of the proposals that are accepted by all citizens and is given by $\cap_{i \in N} A^{i}$. A strategy profile $(p, A)$ is a subgame perfect equilibrium if it induces a Nash equilibrium in every subgame.

A strategy profile $\sigma=\left(\sigma^{i}\right)_{i \in N}$ determines the expected utility $U^{i}(\sigma)$ for each citizen $i$ as evaluated at the beginning of the game. This utility is equal to the expected value of after-tax income. Since strategies are stationary, $U^{i}(\sigma)$ is also the continuation utility of 
citizen $i$, the expected utility as evaluated at the beginning of any bargaining round $r$, and therefore equal to the expected after-tax income at the beginning of any bargaining round $r$.

A strategy profile $(p, A)$ is called a no-delay strategy profile if $p^{i} \in \cap_{j \in N} A^{j}$ for all $i \in N$.

\section{Tax Equilibrium}

The concept of SSPE imposes relatively few restrictions on individual acceptance sets. For instance, it may well happen at equilibrium that a citizen accepts a proposal which is very unfavorable to him in the knowledge that it will be rejected by a citizen that responds next. To avoid such inessential multiplicity we next introduce a more basic notion of equilibrium, called tax equilibrium, which is shown to be essentially equivalent to the notion of SSPE.

Definition 3.1: The profile $p \in X^{N}$ is a tax equilibrium if $p^{i} \in \arg \max _{x \in S} x_{i}$, for all $i \in N$, where $S=\left\{x \in X \mid x \geq \delta \sum_{j \in N}(1 / n) p^{j}\right\}$.

We associate to a tax equilibrium $p$ the strategy profile $\sigma=(p, A(p))$, where

$$
A^{i}(p)=\left\{x \in X \mid x_{i} \geq \delta \sum_{j \in N}(1 / n) p_{i}^{j}\right\}, \quad i \in N .
$$

Notice that at a tax equilibrium, every citizen $i$ makes a proposal $p^{i}$ in the social acceptance set $\cap_{j \in N} A^{j}(p)=\left\{x \in X \mid x \geq \delta \sum_{j \in N}(1 / n) p^{j}\right\}$, which coincides with the set $S$ as defined in Definition 3.1. The strategy profile $(p, A(p))$ therefore satisfies the no-delay property. Observe that $\sum_{j \in N}(1 / n) p^{j}$ is equal to the expected utility in a tax equilibrium. According to the individual acceptance set $A^{i}(p)$, a citizen accepts any proposal that leads to aftertax income greater than or equal to tax equilibrium utility multiplied by $\delta$. Conditional on being the proposer, every citizen makes a proposal that maximizes his income among all the proposals in the social acceptance set. Since all the proposals $p^{j}$ belong to $X$, and $X$ is convex, it also holds that $\sum_{j \in N}(1 / n) p^{j}$ belongs to $X$. Equilibrium utility is non-negative and citizens with higher pre-tax income have higher expected tax equilibrium utility.

Before turning to the existence and uniqueness of tax equilibria in Section 5, we argue first that tax equilibrium is an appropriate concept in the sense that if $p$ is a tax equilibrium, then $(p, A(p))$ is an SSPE.

TheOREM 3.2: If $p \in X^{N}$ is a tax equilibrium, then $(p, A(p))$ is an SSPE. 
Proof: A one-shot deviation in a subgame is a single deviation by the player at the root of the subgame. It holds that $(p, A(p))$ is an SSPE if and only if there is no player having a profitable one-shot deviation. The proof of this fact is standard in the literature and is based on the optimality principle from dynamic programming.

Let $p$ be a tax equilibrium. We demonstrate next that no player has a profitable oneshot deviation from $(p, A(p))$. We denote the tax equilibrium utility by $y=\sum_{j \in N}(1 / n) p^{j}$.

Consider a subgame starting at a history where citizen $i$ is the proposer, so according to $(p, A(p))$ the proposal $p^{i}$ is made by $i$ and next accepted by all players, giving rise to subgame utility $p_{i}^{i}$ for citizen $i$. Consider a one-shot deviation by citizen $i$ to a proposal $x \in X$. If $x$ does not belong to $\cap_{j \in N} A^{j}(p)$, it leads to subgame utility $\delta y_{i}$ for player $i$. Since $\delta y_{i} \leq p_{i}^{i}$ by definition of $p^{i}$, such a deviation is not profitable. If $x$ does belong to $\cap_{j \in N} A^{j}(p)$, it holds that $x_{i} \leq p_{i}^{i}$ by definition of $p^{i}$, and again the deviation is not profitable.

Consider a subgame starting at a history where citizen $i$ is the responder to a proposal $x \in X$. If $x_{j} \geq \delta y_{j}$ for all $j \in N$ such that either $j=i$ or $j$ responds after citizen $i$, then $x$ is accepted, giving subgame utility $x_{i}$ to citizen $i$. A one-shot deviation by citizen $i$ to rejection leads to subgame utility $\delta y_{i} \leq x_{i}$ and is therefore not profitable. If $x_{i}<\delta y_{i}$ and $x_{j} \geq \delta y_{j}$ for all $j \in N$ such that $j$ responds after citizen $i$, then $x$ is rejected and citizen $i$ 's subgame utility is equal to $\delta y_{i}$. A one-shot deviation by citizen $i$ to acceptance leads to subgame utility $x_{i}<\delta y_{i}$ and is therefore not profitable. If $x_{j}<\delta y_{j}$ for some $j \in N$ such that $j$ responds after citizen $i$, then $x$ is rejected and citizen $i$ 's subgame utility is equal to $\delta y_{i}$, irrespective of citizen $i$ 's decision, so one-shot deviations are not profitable. Q.E.D.

Next we argue the converse of Theorem 3.2 to be true. If $(p, A)$ is an SSPE, where $A$ may or may not be equal to $A(p)$, then $p$ is a tax equilibrium.

TheOrem 3.3: If $(p, A)$ is an SSPE, then $(p, A)$ is a no-delay strategy profile, and $p$ is a tax equilibrium.

Proof: Let $(p, A)$ be an SSPE. We denote the SSPE utility by $y$. The proof proceeds in five steps.

(1) $\{x \in X \mid x \gg \delta y\} \subset \cap_{j \in N} A^{j} .^{1}$

Suppose there is $x \in X$ such that $x \gg \delta y$, but $x \notin \cap_{j \in N} A^{j}$. Let player $i$ be such that $x \notin A^{i}$ and for all players $j$ responding after player $i$ it holds that $x \in A^{j}$. Consider a subgame

\footnotetext{
${ }^{1}$ We use the notation $\gg$ to indicate that all components of a first vector strictly exceed the corresponding components of a second vector, whereas $>$ is used to mean that this property holds for at least one component.
} 
starting at a history where citizen $i$ has to respond to proposal $x$. The subgame equilibrium utility of player $i$ is equal to $\delta y_{i}$. A one-shot deviation by citizen $i$ to acceptance leads to subgame utility $x_{i}>\delta y_{i}$ and is therefore profitable, a contradiction.

(2) For all $i \in N, p^{i} \in \cap_{j \in N} A^{j}$.

Suppose by way of contradiction that there is $i \in N$ such that $p^{i} \notin \cap_{j \in N} A^{j}$. Consider the subgame starting at a history where citizen $i$ is the proposer. Since $p^{i}$ is rejected, there is a positive probability that all players receive after-tax income 0 , so $\sum_{j \in N} y_{j}<1$. Moreover, since $y$ is a weighted average of vectors in $X$ and the zero vector, it holds that $y_{j} \leq y_{k}$ if $j<k$. It follows that there is $x \in X$ such that $x \gg y$. The subgame equilibrium utility of player $i$ is equal to $\delta y_{i}$. Consider the one-shot deviation of player $i$ where he proposes $x$. By (1) the proposal $x$ is accepted, leading to subgame utility $x_{i}>\delta y_{i}$ for citizen $i$. We have found a profitable one-shot deviation, a contradiction to $(p, A)$ being an SSPE. We have as a consequence that $y=\sum_{j \in N}(1 / n) p^{j}$.

(3) For all $i \in N, p^{i} \in \arg \max _{x \in \cap_{j \in N} A^{j}} x_{i}$.

Clearly, the availability of a proposal that gives citizen $i$ a strictly higher utility than $p^{i}$ and that is accepted by all citizens leads to a profitable deviation in a subgame starting at a history where citizen $i$ is the proposer.

(4) $\cap_{j \in N} A^{j} \subset S=\{x \in X \mid x \geq \delta y\}$.

Suppose not. Let $x \in \cap_{j \in N} A^{j}$ and $i \in N$ be such that $x_{i}<\delta y_{i}$ and $x_{j} \geq \delta y_{j}$ for all $j \in N$ responding after $i$. Consider the subgame starting at a history where citizen $i$ responds to the proposal $x$. Since $x$ is accepted by $i$ and all his followers, the subgame equilibrium utility of citizen $i$ is $x_{i}$. A one-shot deviation by player $i$ to rejection leads to subgame utility $\delta y_{i}>x_{i}$, and is therefore profitable, a contradiction.

(5) For all $i \in N, p^{i} \in \arg \max _{x \in S} x_{i}$.

By (2) and (4), for all $i \in N, p^{i} \in \cap_{j \in N} A^{j} \subset S$, so $p_{i}^{i} \leq \max _{x \in S} x_{i}$. Suppose there is $i \in N$ and $\bar{x} \in S$ such that $\bar{x}_{i}>p_{i}^{i}$. The vector $\bar{y} \in \mathbb{R}^{N}$ defined by $\bar{y}_{j}=\delta y_{j}+(1-\delta) / n, j \in N$, satisfies $\bar{y} \in S$ and $\bar{y} \gg \delta y$. Let $\bar{z}$ be a strictly convex combination of $\bar{x}$ and $\bar{y}$, so $\bar{z} \in S$ and $\bar{z} \gg \delta y$. For a sufficiently small weight on $\bar{y}$ it holds that $\bar{z}_{i}>p_{i}^{i}$. By (1) it holds that $\bar{z} \in \cap_{j \in N} A^{j}$. We obtain a contradiction to $p^{i}$ being a vector in $\cap_{j \in N} A^{j}$ with maximal component $i$.

Q.E.D.

It follows from Theorem 3.2 and 3.3 that there is no loss of generality to restrict attention to tax equilibria when analyzing the consequences of procedurally fair income 
taxation.

\section{Optimal Proposals}

In this section we characterize the solution to citizen $i$ 's optimization problem of choosing the best proposal within the set of socially acceptable proposals. Given a vector $y \in X$ of equilibrium utilities, citizen $i$ 's optimization problem is

$$
\begin{aligned}
\operatorname{maximize} & x_{i} \\
\text { subject to } & x \in X, \\
& x \geq \delta y .
\end{aligned}
$$

A solution to this problem is denoted $a^{i}(y) \in X$ and yields the proposed after-tax income by citizen $i$ given tax equilibrium utility $y$. In this section we argue this solution to be unique.

The set $\{x \in X \mid x \geq \delta y\}$ is compact. We claim that $\bar{x} \in \mathbb{R}^{n}$ defined by $\bar{x}_{j}=\delta y_{j}$, $j \in[1, n-1]$, and $\bar{x}_{n}=1-\sum_{j \in[1, n-1]} \delta y_{j}$ belongs to $X$, so $X$ is non-empty. Indeed, it holds that $\bar{x}(N)=1$, for every $j \in[1, n-2]$ it holds that $\bar{x}_{j}=\delta y_{j} \leq \delta y_{j+1}=\bar{x}_{j+1}$, and

$$
\bar{x}_{n}=1-\sum_{j \in[1, n-1]} \delta y_{j}>\delta y_{n} \geq \delta y_{n-1}=\bar{x}_{n-1} .
$$

The problem of citizen $i$ therefore involves the maximization of a continuous function on a non-empty compact set and therefore has at least one solution. The following proposition is straightforward to verify and is stated without proof.

Proposition 4.1: Let $a^{i}(y)$ be a solution to the optimization problem of citizen $i \in N$ when tax equilibrium utility is $y \in X$. Then there exists a threshold $k_{i} \in[i, n]$ such that

$$
a_{j}^{i}(y)= \begin{cases}\frac{\delta y\left(\left[i, k_{i}\right]\right)+1-\delta}{k_{i}-i+1}, & j \in\left[i, k_{i}\right], \\ \delta y_{j}, & j \in N \backslash\left[i, k_{i}\right],\end{cases}
$$

and

$$
\delta y_{k_{i}} \leq a_{k_{i}}^{i}(y)<\delta y_{k_{i}+1}
$$

In an optimal proposal by citizen $i$, all citizens in $\left[i, k_{i}\right]$ receive the same after-tax income, equal to $a_{i}^{i}(y)$.

Consider some $y \in X$ and $i \in N$. We define the function $g_{i}:[i, n] \rightarrow \mathbb{R}$ by

$$
g_{i}(k)=\frac{\delta y([i, k])+1-\delta}{k-i+1} .
$$


Then $g_{i}(k)$ is equal to the after-tax income of citizen $i$ when the citizens $j \in N \backslash[i, k]$ are given after-tax income $\delta y_{j}$ and the remainder of aggregate pre-tax income is shared equally between the citizens in $[i, k]$. We define the set

$$
K_{i}=\left\{k \in[i, n] \mid \delta y_{k} \leq g_{i}(k)<\delta y_{k+1}\right\}
$$

From Proposition 4.1 we know that $a_{i}^{i}(y)=g_{i}(k)$ for some $k \in K_{i}$. As Proposition 4.2 below shows, the set $K_{i}$ is a singleton. Therefore, the threshold $k_{i}$ can be characterized as the unique integer $k \in[i, n]$ satisfying the inequality $\delta y_{k} \leq g_{i}(k)<\delta y_{k+1}$.

Proposition 4.2: Consider some $y \in X$ and $i \in N$. The set $K_{i}$ is a singleton.

PROOF: Some elementary algebra shows that for $k \in[i+1, n]$,

$$
g_{i}(k)-g_{i}(k-1)=\frac{\delta y_{k}-g_{i}(k)}{k-i},
$$

SO

$$
g_{i}(k) \geq g_{i}(k-1) \text { if and only if } \delta y_{k} \geq g_{i}(k) .
$$

We show next that $K_{i}$ is an interval. Assume that $j$ and $k$ are elements of $K_{i}$ with $j<k$. We prove that the set $K_{i}$ contains the entire interval $[j, k]$. It holds that

$$
\delta y_{k-1} \leq \delta y_{k} \leq g_{i}(k) \leq g_{i}(k-1)
$$

where the first inequality follows from $y \in X$, the second inequality follows since $k \in K_{i}$, and the third inequality follows from the second inequality and Equation 4.1. Iterating this argument, we obtain the chain of inequalities

$$
\delta y_{j} \leq \cdots \leq \delta y_{k} \leq g_{i}(k) \leq \cdots \leq g_{i}(j)
$$

At the same time, we obtain the chain of inequalities

$$
g_{i}(k) \leq \cdots \leq g_{i}(j)<\delta y_{j+1} \leq \cdots \leq \delta y_{k+1},
$$

where the first tuple of weak inequalities follows from (4.2), the strict inequality since $j \in K_{i}$, and the last tuple of weak inequalities from $y \in X$. The inequalities in (4.2) and (4.3) show that $[j, k]$ is contained in $K_{i}$.

Finally, suppose that both $k-1$ and $k$ belong to the set $K_{i}$. Then $\delta y_{k-1} \leq g_{i}(k-1)<$ $\delta y_{k} \leq g_{i}(k)<\delta y_{k+1}$, thus in particular $g_{i}(k-1)<g_{i}(k)$. But this is in contradiction to Inequality 4.1 since $g_{i}(k) \geq \delta y_{k}$ implies that $g_{i}(k-1) \geq g_{i}(k)$. This proves that $K_{i}$ is a 
singleton.

Q.E.D.

Combining Proposition 4.1 and 4.2 leads to the following result.

Corollary 4.3: The solution $a^{i}(y)$ to the optimization problem of citizen $i \in N$ is unique for every $y \in X$.

Proposition 4.4: For every citizen $i \in N$, the function $a^{i}: X \rightarrow X$ is continuous.

Proof: The correspondence $\varphi: X \rightarrow X$ defined by

$$
\varphi(y)=\{x \in X \mid x \geq \delta y\}, \quad y \in X
$$

is compact-valued and has a closed graph, so is upper hemi-continuous. To show it is lower hemi-continuous, consider $\bar{y} \in X$, a sequence $\left(y_{m}\right)_{m \in \mathbb{N}}$ in $X$ converging to $\bar{y}$, and $\bar{x} \in \varphi(\bar{y})$. We have to construct a sequence $\left(x_{m}\right)_{m \in \mathbb{N}}$ in $X$ such that $x_{m} \in \varphi\left(y_{m}\right)$ for all $m \in \mathbb{N}$, and $x_{m} \rightarrow \bar{x}$. We define

$$
x_{m}=\delta y_{m}+\alpha_{m} \max \left\{\bar{x}-\delta y_{m}, 0\right\},
$$

where

$$
\alpha_{m}=\frac{1-\delta}{\sum_{j \in N} \max \left\{\bar{x}_{j}-\delta y_{m, j}, 0\right\}}
$$

and $\max \left\{\bar{x}-\delta y_{m}, 0\right\}$ denotes the vector obtained by taking the component-wise maximum. The denominator in the expression for $\alpha_{m}$ is well-defined since

$$
\sum_{j \in N} \max \left\{\bar{x}_{j}-\delta y_{m, j}, 0\right\} \geq \sum_{j \in N}\left(\bar{x}_{j}-\delta y_{m, j}\right)=1-\delta
$$

so $\alpha_{m} \in(0,1]$.

We show next that for all $m \in \mathbb{N}$ it holds that $x_{m} \in \varphi\left(y_{m}\right)$. Clearly it holds that $x_{m}(N)=1$ and $x_{m} \geq \delta y_{m}$. Consider some $i \in[1, n-1]$. If $\bar{x}_{i} \leq \delta y_{m, i}$, then $x_{m, i}=\delta y_{m, i} \leq$ $\delta y_{m, i+1} \leq x_{m, i+1}$. Consider the case where $\bar{x}_{i}>\delta y_{m, i}$, so $x_{m, i}=\alpha_{m} \bar{x}_{i}+\left(1-\alpha_{m}\right) \delta y_{m, i}$. If $\bar{x}_{i+1} \leq \delta y_{m, i+1}$, then

$$
x_{m, i+1}=\delta y_{m, i+1} \geq \bar{x}_{i+1} \geq \bar{x}_{i}>\alpha_{m} \bar{x}_{i}+\left(1-\alpha_{m}\right) \delta y_{m, i}=x_{m, i} .
$$

If $\bar{x}_{i+1}>\delta y_{m, i+1}$, then

$$
x_{m, i+1}=\alpha_{m} \bar{x}_{i+1}+\left(1-\alpha_{m}\right) \delta y_{m, i+1} \geq \alpha_{m} \bar{x}_{i}+\left(1-\alpha_{m}\right) \delta y_{m, i}=x_{m, i} .
$$


Since $y_{m} \rightarrow \bar{y}$, we have that

$$
\alpha_{m}=\frac{1-\delta}{\sum_{j \in N} \max \left\{\bar{x}_{j}-\delta y_{m, j}, 0\right\}} \rightarrow \frac{1-\delta}{\sum_{j \in N} \max \left\{\bar{x}_{j}-\delta \bar{y}_{j}, 0\right\}}=\frac{1-\delta}{\sum_{j \in N}\left(\bar{x}_{j}-\delta \bar{y}_{j}\right)}=1,
$$

SO

$$
x_{m} \rightarrow \delta \bar{y}+\max \{\bar{x}-\delta \bar{y}, 0\}=\delta \bar{y}+\bar{x}-\delta \bar{y}=\bar{x} .
$$

We have shown that $\varphi$ is lower hemi-continuous.

The function $f: X \rightarrow \mathbb{R}$ defined by $f(x)=x_{i}$ is continuous. An application of the maximum theorem yields that the correspondence $\mu: X \rightarrow X$ defined by

$$
\mu(y)=\{\bar{x} \in \varphi(y) \mid \forall x \in \varphi(y), f(x) \leq f(\bar{x})\}, \quad y \in X
$$

is upper hemi-continuous. Since $\mu(y)=\left\{a^{i}(y)\right\}$ for all $y \in X$, it follows that $a^{i}$ is a continuous function.

Q.E.D.

\section{Uniqueness of the Tax Equilibrium}

Very little is known about the uniqueness of SSPE in multi-lateral bargaining models with unanimous agreement. It follows from the results in Kalandrakis (2006) that the most one can hope for is that the number of equilibria is odd for generic specifications of the model. Under more special assumptions it is possible to obtain uniqueness results, as is the case in Merlo and Wilson (1995). There it is assumed that the set of feasible utilities is generated by the division of a physical cake $C=\left\{c \in \mathbb{R}_{+}^{n} \mid \sum_{i=1}^{n} c_{i} \leq 1\right\}$, with player $i$ deriving utility $u^{i}\left(c_{i}\right)$ from a share $c_{i}$, where $u^{i}(0)=0 .{ }^{2}$ For the model with random proposers, as studied in this paper, Merlo and Wilson (1995) show that equilibria are unique when the utility functions are all linear. For the model with deterministic proposers, it is sufficient that the utility functions are concave. They also provide a more abstract uniqueness result. However, our set $X$ does not lead to continuous functions $\xi_{i}$ as required in their Assumption (A1), neither can it be derived from an underlying physical cake as required in their Assumption (A2) because of our requirement that $x_{i} \leq x_{i+1}$ for $x \in X$. Other uniqueness results are given in Imai and Salonen (2000), Cho and Duggan (2003), and Cardona and Ponsatí (2007) for the case where the physical cake $C$ is a unit interval. Imai and Salonen (2000) study the case where utility functions are either monotonically increasing or monotonically decreasing on the unit interval. Cho and Duggan (2003) prove uniqueness for quadratic utility functions, and show that uniqueness does not

\footnotetext{
${ }^{2}$ The Merlo and Wilson (1995) set-up allows the size of the physical cake $C$ to be state-dependent, rather than being fixed to 1 .
} 
hold for general concave utility functions. It follows from Cardona and Ponsatí (2007) that concavity together with symmetry around the peak is sufficient for uniqueness. Obviously, none of these results implies uniqueness in our setting since the multi-dimensional set $X$ cannot be generated from a 1-dimensional physical cake $C$ by utility functions satisfying the assumptions of the various uniqueness results.

In this section, we demonstrate that the tax equilibrium is unique by establishing that the function $f: X \rightarrow X$ defined by

$$
f(y)=\sum_{i=1}^{n} \frac{1}{n} a^{i}(y), \quad y \in X,
$$

is a contraction with contraction coefficient $\delta$, i.e. for all $y, \bar{y} \in X$ it holds that $\| f(y)-$ $f(\bar{y})\|\leq \delta\| y-\bar{y} \|$, where $\|\cdot\|$ denotes the infinity norm. It follows directly from the definition of a tax equilibrium that $y \in X$ is the tax equilibrium utility if and only if $y$ is a fixed point of $f$. In this case $a^{i}(y)$ is the equilibrium proposal for after-tax income by citizen $i \in N$.

Consider a citizen $i \in N$. We use the notation $k_{i}(y)$ for the unique threshold as defined in Proposition 4.1, so we make the dependence on $y \in X$ explicit. For $k \in[i, n]$ we define $X_{k}^{i}=\left\{y \in X \mid k_{i}(y)=k\right\}$. On the set $X_{k}^{i}$, the function $a^{i}$ is given by

$$
a_{j}^{i}(y)= \begin{cases}\frac{\delta y([i, k])+1-\delta}{k-i+1}, & j \in[i, k], \\ \delta y_{j}, & j \in N \backslash[i, k] .\end{cases}
$$

We denote the closure of $X_{k}^{i}$ by $\bar{X}_{k}^{i}$. The closed-form expression for $\bar{X}_{k}^{i}$ is given by

$$
\bar{X}_{k}^{i}=\left\{y \in X \mid \delta y_{k} \leq \frac{\delta y[i, k]+1-\delta}{k-i+1} \leq \delta y_{k+1}\right\} .
$$

Proposition 5.1: For all $i \in N$, for all $k \in[i, n]$, the function $a^{i}$ is a contraction on the set $\bar{X}_{k}^{i}$ with contraction coefficient $\delta$.

Proof: We first argue $a^{i}$ to be a contraction on the set $X_{k}^{i}$ with contraction coefficient $\delta$. Take points $y$ and $\bar{y}$ in $X_{k}^{i}$. For each $j \in[i, k]$ we have the inequalities

$$
\begin{aligned}
\left|a_{j}^{i}(y)-a_{j}^{i}(\bar{y})\right| & =\left|\frac{\delta y([i, k])+1-\delta}{k-i+1}-\frac{\delta \bar{y}([i, k])+1-\delta}{k-i+1}\right| \\
& =\frac{\delta}{k-i+1}|y([i, k])-\bar{y}([i, k])| \\
& \leq \frac{\delta}{k-i+1} \sum_{j=i}^{k}\left|y_{j}-\bar{y}_{j}\right| \leq \frac{\delta}{k-i+1} \sum_{j=i}^{k}\|y-\bar{y}\|=\delta\|y-\bar{y}\| .
\end{aligned}
$$


For each $j \in N \backslash[i, k]$ we have

$$
\left|a_{j}^{i}(y)-a_{j}^{i}(\bar{y})\right|=\delta\left|y_{j}-\bar{y}_{j}\right| \leq \delta\|y-\bar{y}\|
$$

We conclude that

$$
\left\|a^{i}(y)-a^{i}(\bar{y})\right\| \leq \delta\|y-\bar{y}\|
$$

Now take points $y$ and $\bar{y}$ in $\bar{X}_{k}^{i}$. Let $\left(y_{n}\right)_{n \in \mathbb{N}}$ and $\left(\bar{y}_{n}\right)_{n \in \mathbb{N}}$ be sequences in $X_{k}^{i}$ converging to $y$ and $\bar{y}$, respectively. Then, for all $n \in \mathbb{N}$,

$$
\left\|a^{i}\left(y_{n}\right)-a^{i}\left(\bar{y}_{n}\right)\right\| \leq \delta\left\|y_{n}-\bar{y}_{n}\right\|
$$

and by taking the limit as $n \rightarrow \infty$, we have

$$
\left\|a^{i}(y)-a^{i}(\bar{y})\right\| \leq \delta\|y-\bar{y}\|
$$

where we use the continuity of $a^{i}$ as derived in Proposition 4.4.

Q.E.D.

The next step is to extend the result of Proposition 5.1, claiming that $a^{i}$ is a contraction on each set $\bar{X}_{k}^{i}$, to a result valid for the entire domain $X$. To derive this extension, we exploit the fact that each $\bar{X}_{k}^{i}$ is a polytope.

THEOREM 5.2: For all $i \in N$, the function $a^{i}$ is a contraction with contraction coefficient $\delta$.

Proof: It follows from Proposition 4.2 that the sets $X_{i}^{i}, \ldots, X_{n}^{i}$ partition the set $X$, so the sets $\bar{X}_{i}^{i}, \ldots, \bar{X}_{n}^{i}$ form a covering of $X$. Since all the equalities and inequalities defining $\bar{X}_{k}^{i}$ are linear, it follows that $\bar{X}_{k}^{i}$ is a polytope.

Take points $y$ and $\bar{y}$ in $X$ and consider a straight line from $y$ to $\bar{y}$. Since all the sets $\bar{X}_{k}^{i}$ are polytopes, there exist $y_{1}, \ldots, y_{m}$ which are all on the line from $y$ to $\bar{y}$ and which are such that $y_{1}=y, y_{m}=\bar{y}$, and for all $\ell \in[1, m-1], y_{\ell}, y_{\ell+1} \in \bar{X}_{k}^{i}$ for some $k \in[i, n]$. We have that

$$
\left\|a^{i}(y)-a^{i}(\bar{y})\right\| \leq \sum_{\ell=1}^{m-1}\left\|a^{i}\left(y_{\ell}\right)-a^{i}\left(y_{\ell+1}\right)\right\| \leq \sum_{\ell=1}^{m-1} \delta\left\|y_{\ell}-y_{\ell+1}\right\|=\delta\|y-\bar{y}\|,
$$

where the second inequality follows from Proposition 5.1.

THEOREM 5.3: The function $f$ is a contraction with contraction coefficient $\delta$. 


\begin{tabular}{|cc|}
\hline$a^{1}(y)$ & $a^{2}(y)$ \\
\hline $1-\delta+\delta y_{1}$ & $\delta y_{1}$ \\
$\delta y_{2}$ & $1-\delta+\delta y_{2}$ \\
\hline
\end{tabular}

Table 1: The functions $a^{i}$ if $k_{1}=1$ and $k_{2}=2$.

Proof: For each $y$ and $\bar{y}$ we have the following inequalities

$$
\|f(y)-f(\bar{y})\|=\left\|\sum_{i=1}^{n} \frac{1}{n}\left(a^{i}(y)-a^{i}(\bar{y})\right)\right\| \leq \sum_{i=1}^{n} \frac{1}{n}\left\|a^{i}(y)-a^{i}(\bar{y})\right\| \leq \delta\|y-\bar{y}\|,
$$

where the last inequality follows from Theorem 5.2.

Q.E.D.

The function $f$ being a contraction implies that it has a unique fixed point. Since the fixed points of $f$ are in a one-one correspondence with tax equilibria, we find the following corollary.

COROLlary 5.4: There is a unique tax equilibrium.

\section{Two Special Cases}

This section calculates the tax equilibrium for the case where society consists of two income groups and the case where it consists of three income groups. This analysis also gives some first insights into the equilibrium choice of $k_{i}$ as well as the limit taxation scheme when $\delta \uparrow 1$.

\subsection{Two Players}

In this subsection we assume $n=2$. We compute the values of $\delta$ for which in a tax equilibrium it holds that $k_{1}=1$ and $k_{2}=2$, where the latter equality follows by definition.

Table 1 shows the functions $a^{i}$. At equilibrium it holds that $a^{1}(y)+a^{2}(y)=2 y$, which can be rewritten as

$$
\left[\begin{array}{cc}
2-2 \delta & 0 \\
0 & 2-2 \delta
\end{array}\right]\left[\begin{array}{l}
y_{1} \\
y_{2}
\end{array}\right]=\left[\begin{array}{l}
1-\delta \\
1-\delta
\end{array}\right]
$$

Solving it gives $y_{1}=y_{2}=1 / 2$.

Moreover, $k_{i}$ should satisfy the inequalities presented in Proposition 4.1 , so $\delta y_{1} \leq$ $a_{1}^{1}(y)<\delta y_{2}$ and $\delta y_{2} \leq a_{2}^{2}(y)$. It holds that $a_{1}^{1}(y)=1-\delta / 2>\delta / 2=\delta y_{2}$, so there is no value for $\delta$ for which $k_{1}=1$ and $k_{2}=2$. 


\begin{tabular}{|cc|}
\hline$a^{1}(y)$ & $a^{2}(y)$ \\
\hline$\frac{1}{2}$ & $\delta y_{1}$ \\
$\frac{1}{2}$ & $1-\delta+\delta y_{2}$ \\
\hline
\end{tabular}

Table 2: The functions $a^{i}$ if $k_{1}=2$ and $k_{2}=2$.

\begin{tabular}{|cc|c|}
\hline$p^{1}$ & $p^{2}$ & $y$ \\
\hline$\frac{1}{2}$ & $\frac{\delta}{4-2 \delta}$ & $\frac{1}{4-2 \delta}$ \\
$\frac{1}{2}$ & $\frac{4-3 \delta}{4-2 \delta}$ & $\frac{3-2 \delta}{4-2 \delta}$ \\
\hline
\end{tabular}

Table 3: The tax equilibrium proposals $p^{i}$ and tax equilibrium utility $y$ if $k_{1}=k_{2}=2$.

Since a tax equilibrium exists by Corollary 5.4, we have for all values of $\delta$ in equilibrium $k_{1}=2$ and $k_{2}=2$. Table 2 depicts the corresponding functions $a^{i}$.

At equilibrium it holds that $a^{1}(y)+a^{2}(y)=2 y$, which can be rewritten as

$$
\left[\begin{array}{cc}
2-\delta & 0 \\
0 & 2-\delta
\end{array}\right]\left[\begin{array}{l}
y_{1} \\
y_{2}
\end{array}\right]=\left[\begin{array}{c}
\frac{1}{2} \\
\frac{3}{2}-\delta
\end{array}\right]
$$

Solving it gives $y_{1}=1 /(4-2 \delta)$ and $y_{2}=(3-2 \delta) /(4-2 \delta)$. It can be verified that $k_{1}$ and $k_{2}$ satisfy the inequalities of Proposition 4.1 for all values of $\delta$. Table 3 presents the tax equilibrium proposals and the tax equilibrium utility.

We are interested in the limit of tax equilibrium proposals and tax equilibrium utility when $\delta$ tends to 1 from below. Citizen 1 always proposes to split income equally, irrespective of the value of $\delta$. Citizen 2 does the same in the limit, since

$$
\lim _{\delta \uparrow 1} \frac{\delta}{4-2 \delta}=\frac{1}{2} \text { and } \lim _{\delta \uparrow 1} \frac{4-3 \delta}{4-2 \delta}=\frac{1}{2} .
$$

It then follows that in the limit, tax equilibrium utility as well as tax equilibrium after-tax income is equal to $1 / 2$ for both citizens.

\subsection{Three Players}

We now analyze the case where $n=3$. We compute the values of $\delta$ for which in equilibrium $k_{1}=k_{2}=k_{3}=3$. We have the following system of 12 equations and 12 unknowns.

$$
\begin{aligned}
p^{i} & =a^{i}(y), \quad i \in[1,3], \\
y & =\sum_{i \in[1,3]} \frac{1}{n} p^{i},
\end{aligned}
$$

which we can simplify as the following system in 3 equations and 3 unknowns:

$$
a^{1}(y)+a^{2}(y)+a^{3}(y)=3 y .
$$




\begin{tabular}{|ccc|c|}
\hline$p^{1}$ & $p^{2}$ & $p^{3}$ & $y$ \\
\hline$\frac{1}{3}$ & $\frac{\delta}{3(3-2 \delta)}$ & $\frac{\delta}{3(3-2 \delta)}$ & $\frac{1}{3(3-2 \delta)}$ \\
$\frac{1}{3}$ & $\frac{9-7 \delta}{6(3-2 \delta)}$ & $\frac{\delta(15-11 \delta)}{6(3-\delta)(3-2 \delta)}$ & $\frac{15-11 \delta}{6(3-\delta)(3-2 \delta)}$ \\
$\frac{1}{3}$ & $\frac{9-7 \delta}{6(3-2 \delta)}$ & $\frac{25 \delta^{2}-75 \delta+54}{6(3-\delta)(3-2 \delta)}$ & $\frac{12 \delta^{2}-41 \delta+33}{6(3-\delta)(3-2 \delta)}$ \\
\hline
\end{tabular}

Table 4: The tax equilibrium proposals $p^{i}$ and tax equilibrium utility $y$ if $k_{1}=k_{2}=k_{3}=3$.

\begin{tabular}{|ccc|c|}
\hline$p^{1}$ & $p^{2}$ & $p^{3}$ & $y$ \\
\hline$\frac{9-12 \delta+4 \delta^{2}}{18-15 \delta}$ & $\frac{3 \delta-2 \delta^{2}}{18-15 \delta}$ & $\frac{3-2 \delta^{2}}{18-15 \delta}$ & $\frac{3-2 \delta}{18-15 \delta}$ \\
$\frac{9-12 \delta+4 \delta^{2}}{18-15 \delta}$ & $\frac{9-9 \delta+\delta^{2}}{18-15 \delta}$ & $\frac{\delta}{3}$ & $\frac{1}{3}$ \\
$\frac{9 \delta-8 \delta^{2}}{18-15 \delta}$ & $\frac{9-9 \delta+\delta^{2}}{18-15 \delta}$ & $\frac{18-24 \delta+7 \delta^{2}}{18-15 \delta}$ & $\frac{9-8 \delta}{18-15 \delta}$ \\
\hline
\end{tabular}

Table 5: The tax equilibrium proposals $p^{i}$ and equilibrium utility $y$ if $k_{1}=2, k_{2}=k_{3}=3$.

This system can be rewritten as

$$
\left[\begin{array}{ccc}
3-2 \delta & 0 & 0 \\
0 & 3-\frac{3}{2} \delta & -\frac{1}{2} \delta \\
0 & -\frac{1}{2} \delta & 3-\frac{3}{2} \delta
\end{array}\right]\left[\begin{array}{c}
y_{1} \\
y_{2} \\
y_{3}
\end{array}\right]=\left[\begin{array}{c}
\frac{1}{3} \\
\frac{5}{6}-\frac{1}{2} \delta \\
\frac{11}{6}-\frac{3}{2} \delta
\end{array}\right]
$$

or equivalently

$$
\left[\begin{array}{ccc}
18-12 \delta & 0 & 0 \\
0 & 18-9 \delta & -3 \delta \\
0 & -3 \delta & 18-9 \delta
\end{array}\right]\left[\begin{array}{l}
y_{1} \\
y_{2} \\
y_{3}
\end{array}\right]=\left[\begin{array}{c}
2 \\
5-3 \delta \\
11-9 \delta
\end{array}\right]
$$

Solving the system we obtain the unique solution given by the last column of Table 4 . To check that the proposals in Table 4 constitute an equilibrium, one has to verify the inequalities $\delta y_{3} \leq p_{3}^{1}, \delta y_{3} \leq p_{3}^{2}$, and $\delta y_{3} \leq p_{3}^{3}$. The most stringent inequality is $\delta y_{3} \leq p_{3}^{1}$. It holds if and only if $(\delta-3 / 4)(\delta-1)(\delta-2) \leq 0$, which is the case for $\delta \in[0,3 / 4]$.

We now compute the values of $\delta$ for which in equilibrium $k_{1}=2, k_{2}=3$, and $k_{3}=3$. The system of equations $6 y-2 a^{1}(y)-2 a^{2}(y)-2 a^{3}(y)=0$ can be rewritten as

$$
\left[\begin{array}{ccc}
6-5 \delta & -\delta & 0 \\
-\delta & 6-4 \delta & -\delta \\
0 & -\delta & 6-5 \delta
\end{array}\right]\left[\begin{array}{l}
y_{1} \\
y_{2} \\
y_{3}
\end{array}\right]=\left[\begin{array}{c}
1-\delta \\
2(1-\delta) \\
3(1-\delta)
\end{array}\right]
$$

Table 5 presents the solution. To check that the proposals in Table 5 constitute an equilibrium, one has to verify the inequalities $\delta y_{2} \leq p_{2}^{1}<\delta y_{3}, \delta y_{3} \leq p_{3}^{2}$, and $\delta y_{3}^{1} \leq p_{3}^{3}$. The first three inequalities are respectively equivalent to $(\delta-1)^{2} \geq 0,(\delta-1)(4 \delta-3)<0$, 
and $\delta-1 \leq 0$, whereas the fourth inequality is satisfied for all values of $\delta$. It follows that $\delta \in(3 / 4,1)$ gives rise to a tax equilibrium with $k_{1}=2, k_{2}=3$, and $k_{3}=3$.

For the case with $n=3$, the choice of $k_{i}$ depends on the value of $\delta$. It is not true that for $\delta$ close to $1, k_{i}=3$ for all citizens $i$. Nevertheless, we verify next that in the limit all players propose to share the aggregate income equally. Indeed,

$$
\begin{aligned}
& \lim _{\delta \uparrow 1}\left(\frac{9-12 \delta+4 \delta^{2}}{18-15 \delta}, \frac{9-12 \delta+4 \delta^{2}}{18-15 \delta}, \frac{9 \delta-8 \delta^{2}}{18-15 \delta}\right)=\left(\frac{1}{3}, \frac{1}{3}, \frac{1}{3}\right), \\
& \lim _{\delta \uparrow 1}\left(\frac{3 \delta-2 \delta^{2}}{18-15 \delta}, \frac{9-9 \delta+\delta^{2}}{18-15 \delta}, \frac{9-9 \delta+\delta^{2}}{18-15 \delta}\right)=\left(\frac{1}{3}, \frac{1}{3}, \frac{1}{3}\right), \\
& \lim _{\delta \uparrow 1}\left(\frac{3-2 \delta^{2}}{18-15 \delta}, \frac{\delta}{3}, \frac{18-24 \delta+7 \delta^{2}}{18-15 \delta}\right)=\left(\frac{1}{3}, \frac{1}{3}, \frac{1}{3}\right) .
\end{aligned}
$$

As a consequence, the tax equilibrium expected utility as well as the tax equilibrium expected after-tax income is equal to $1 / 3$ for all citizens.

\section{Procedurally Fair Taxation}

In this section we make a first analysis of the limit of tax equilibria when $\delta \uparrow 1$ for an arbitrary number of players. A first result is that all citizens make the same proposal in the limit.

THEOREM 7.1: Let $\left(\delta_{m}\right)_{m \in \mathbb{N}}$ be a sequence of continuation probabilities converging to 1 and, for $m \in \mathbb{N}$, let $p_{m}$ be the tax equilibrium corresponding to continuation probability $\delta_{m}$. Then for any $i, j \in N$ it holds that $\left\|p_{m}^{i}-p_{m}^{j}\right\| \rightarrow 0$.

Proof: Let $y_{m}$ be the tax equilibrium utility corresponding to $p_{m}$. Since $p_{m}^{i} \geq \delta_{m} y_{m}$ and $\sum_{k=1}^{n} y_{m, k}=1$, we have that $p_{m, k}^{i} \leq \delta_{m} y_{m, k}+\left(1-\delta_{m}\right)$ for all $k \in N$. Therefore,

$$
\left\|p_{m}^{i}-p_{m}^{j}\right\|=\max _{k \in N}\left|p_{m, k}^{i}-p_{m, k}^{j}\right| \leq 1-\delta_{m} .
$$

By taking the limit as $m$ goes to infinity, the result follows.

Q.E.D.

We consider after-tax incomes to be procedurally fair if they are the limit of after-tax incomes proposed in the tax equilibrium when $\delta$ converges to one.

Definition 7.2: After-tax incomes $x$ are procedurally fair if they are the limit of a sequence $\left(p_{m}^{i}\right)_{m \in \mathbb{N}}$, where $p_{m}^{i}$ is the proposal of some citizen $i \in N$ in the tax equilibrium corresponding to $\delta_{m}$ and $\left(\delta_{m}\right)_{m \in \mathbb{N}}$ is a sequence of continuation probabilities converging to one.

When studying procedurally fair after-tax incomes, the choice of the proposer $i$ is irrelevant by Theorem 7.1. 
We are interested in two questions. First, are procedurally fair after-tax incomes uniquely determined? And if so, how are they characterized? The results in Section 6 show that when $n=2$ or $n=3$, procedurally fair after-tax incomes are uniquely determined and equal to the egalitarian solution. However, demonstrating such a result for higher values of $n$ requires techniques different from brute force calculations.

The literature on multi-lateral bargaining with unanimous agreement has obtained a number of results on the convergence of non-cooperative equilibrium outcomes to the Nash bargaining solution. In particular, such results have been demonstrated in Hart and MasColell (1996), Laruelle and Valenciano (2007), Miyakawa (2008), Kultti and Vartiainen (2010), and Britz, Herings and Predtetchinski (2010) under increasingly weaker conditions.

Definition 7.3 The Nash product is the function $\rho: X \rightarrow \mathbb{R}$ defined by

$$
\rho(x)=\prod_{i \in N} x_{i}, \quad x \in X
$$

The Nash bargaining solution is the unique maximizer of the function $\rho$ on the set $X$.

The following result establishes that the Nash bargaining solution indeed corresponds to the egalitarian income distribution. The vector $e$ denotes the $n$-dimensional vector of ones.

THEOREM 7.4 The Nash bargaining solution is equal to $(1 / n)$ e.

Proof: The Nash bargaining solution, say $x^{*}$, allocates a positive amount of income to citizen 1. Indeed, allocating zero income to citizen 1 leads to a Nash product of zero, whereas the egalitarian solution belongs to $X$ and leads to a strictly positive Nash product. By definition of $X$, allocating a positive after-tax income to citizen 1 implies allocating a positive after-tax income to all citizens.

It therefore holds that $x^{*}$ is a solution to the program

$$
\begin{aligned}
\operatorname{maximize} & \sum_{i=1}^{n} \frac{1}{n} \ln \left(x_{i}\right) \\
\text { subject to } & \\
& \sum_{i=1}^{n} x_{i}=1, \\
& x_{i} \leq x_{i+1}, \quad i \in[1, n-1] .
\end{aligned}
$$

The Lagrangian is given by

$$
\mathcal{L}=\sum_{i=1}^{n} \frac{1}{n} \ln \left(x_{i}\right)-\lambda\left(\sum_{i=1}^{n} x_{i}-1\right)-\sum_{i=1}^{n-1} \mu_{i}\left(x_{i}-x_{i+1}\right) .
$$


A solution $\left(\lambda^{*}, \mu^{*}, x^{*}\right)$ to the optimization problem is therefore characterized by

$$
\begin{array}{ll}
\frac{1}{n x_{i}^{*}}-\lambda^{*}-\mu_{i}^{*}+\mu_{i-1}^{*}=0, & i \in[1, n], \\
\sum_{i=1}^{n} x_{i}^{*}=1, & i \in[1, n-1], \\
x_{i}^{*} \leq x_{i+1}^{*}, & i \in[1, n-1], \\
\mu_{i}^{*}\left(x_{i}^{*}-x_{i+1}^{*}\right)=0, & i \in[1, n-1], \\
\mu_{i}^{*} \geq 0, &
\end{array}
$$

where $\mu_{0}^{*}$ and $\mu_{n}^{*}$ are equal to 0 by definition. It is immediately verified that $\lambda^{*}=1, \mu^{*}=0$, and $x^{*}=(1 / n) e$ constitutes a solution.

Q.E.D.

We have found that the Nash bargaining solution coincides with the egalitarian solution for all values of $n$. Unfortunately, we cannot yet conclude that procedurally fair after-tax incomes are equal to those of the egalitarian solution. The reason is that all the results on convergence of multi-lateral bargaining with unanimous agreement to the Nash bargaining solution assume that the set of feasible utilities has a differentiable Pareto frontier, a requirement obviously not met by $X$.

Kultti and Vartiainen (2010) provide an example showing that without differentiability the unique bargaining equilibrium may not converge to the Nash bargaining solution. Herings and Predtetchinski (2011a) show that without differentiability the limit of bargaining

equilibria may not be unique. For instance, for a set $\tilde{X}$ given by the intersection of two halfspaces,

$$
\tilde{X}=\left\{x \in \mathbb{R}^{3} \mid 2 x_{1}+x_{2}+3 x_{3} \leq 15\right\} \cap\left\{x \in \mathbb{R}^{3} \mid x_{1}+2 x_{2}+3 x_{3} \leq 15\right\},
$$

Herings and Predtetchinski (2011a) show that bargaining equilibria converge to $(3,3,2)$, whereas the Nash bargaining solution is given by $(10 / 3,10 / 3,5 / 3)$.

\section{Convergence to the Nash Bargaining Solution}

In this section we will argue that irrespective of the number of citizens, the only procedurally fair after-tax incomes correspond to the egalitarian solution, and therefore to the Nash bargaining solution by Theorem 7.4. The first result of this section claims that tax equilibrium utility is strictly increasing in pre-tax income.

THEOREM 8.1: Let $p \in X^{N}$ be a tax equilibrium with utility $y$. Then it holds that $y_{1}<y_{2}<\cdots<y_{n}$. 
Proof: Since $y \in X$, we have that $y_{1} \leq y_{2} \leq \cdots \leq y_{n}$. Consider some $i \in[1, n-1]$. We show that $y_{i}<y_{i+1}$. It holds that

$$
p_{i+1}^{i+1}=\frac{\delta y\left(\left[i+1, k_{i+1}\right]\right)+1-\delta}{k_{i+1}-i} \geq \frac{1-\delta}{k_{i+1}-i}+\delta y_{i}>\delta y_{i}=p_{i}^{i+1},
$$

where the weak inequality uses that the average of $y_{i+1}, \ldots, y_{k_{i+1}}$ is at least $y_{i+1} \geq y_{i}$. Since $p^{j} \in X$ for all $j \in N$, it holds that $p_{i}^{j} \leq p_{i+1}^{j}$ for all $j \in N$. Moreover, by (??) it holds that $p_{i}^{i+1}<p_{i+1}^{i+1}$. Since $y=\sum_{j \in N}(1 / n) p^{j}$, we find that $y_{i}<y_{i+1}$.

Q.E.D.

The next result claims that the thresholds $k_{i}$ are weakly increasing in $i$.

TheOREM 8.2: Let $p \in X^{N}$ be a tax equilibrium with thresholds $k_{i}, i \in N$. Then it holds that $k_{1} \leq k_{2} \leq \cdots \leq k_{n}$.

Proof: Consider some $i \in[1, n-1]$. We show that $k_{i} \leq k_{i+1}$.

If $k_{i}=i$, then obviously $k_{i}<i+1 \leq k_{i+1}$. Consider the case where $k_{i}>i$. Then

$$
\begin{aligned}
a_{k_{i}}^{i}(y) & =\frac{\delta y\left(\left[i, k_{i}\right]\right)+1-\delta}{k_{i}-i+1}=\frac{\delta y\left(\left[i, k_{i}\right]\right)}{k_{i}-i+1}+\frac{1-\delta}{k_{i}-i+1} \\
& <\frac{\delta y\left(\left[i+1, k_{i}\right]\right)}{k_{i}-i}+\frac{1-\delta}{k_{i}-i}=\frac{\delta y\left(\left[i+1, k_{i}\right]\right)+1-\delta}{k_{i}-i}=g_{i+1}\left(k_{i}\right),
\end{aligned}
$$

where the inequality follows from the fact that $y_{i}<\cdots<y_{k_{i}}$ as shown in Theorem 8.1. We have that $\delta y_{k_{i}} \leq a_{k_{i}}^{i}(y)<g_{i+1}\left(k_{i}\right)$, and therefore $k_{i+1} \geq k_{i}$.

We show next that any citizen $i$ that does not have the highest income, proposes the same income to citizen $i+1$ as to himself. This property can be verified in Section 6 to hold for $n=2$ and $n=3$.

TheOREM 8.3: Let $p \in X^{N}$ be a tax equilibrium with thresholds $k_{i}, i \in N$. Then, for every $i \in[1, n-1]$, it holds that $k_{i}>i$.

Proof: Tax equilibrium utility $y$ should satisfy $y=\sum_{i \in N}(1 / n) a^{i}(y)$, with $a^{i}(y)$ given by the expression in Proposition 4.1.

Suppose $k_{i^{\prime}}=i^{\prime}$ for some $i^{\prime} \in[1, n-1]$. For $i>i^{\prime}$ it holds that

$$
\sum_{j \in\left[1, i^{\prime}\right]} a_{j}^{i}(y)=\sum_{j \in\left[1, i^{\prime}\right]} \delta y_{j} .
$$

For $i \leq i^{\prime}$ it holds that

$$
\sum_{j \in\left[1, i^{\prime}\right]} a_{j}^{i}(y)=\sum_{j \in\left[1, i^{\prime}\right]} \delta y_{j}+(1-\delta),
$$


where we use that $k_{i^{\prime}}=i^{\prime}$ by our supposition and $k_{i} \leq k_{i^{\prime}}=i^{\prime}$ for $i \leq i^{\prime}$ by Theorem 8.2. We have that

$$
\begin{aligned}
\sum_{j \in\left[1, i^{\prime}\right]} y_{j} & =\sum_{j \in\left[1, i^{\prime}\right]} \sum_{i \in N} \frac{1}{n} a_{j}^{i}(y) \\
& =\frac{1}{n} \sum_{i \in\left[1, i^{\prime}\right]} \sum_{j \in\left[1, i^{\prime}\right]} a_{j}^{i}(y)+\frac{1}{n} \sum_{i \in\left[i^{\prime}+1, n\right]} \sum_{j \in\left[1, i^{\prime}\right]} a_{j}^{i}(y) \\
& =\frac{i^{\prime}}{n} \sum_{j \in\left[1, i^{\prime}\right]} \delta y_{j}+\frac{i^{\prime}}{n}(1-\delta)+\frac{n-i^{\prime}}{n} \sum_{j \in\left[1, i^{\prime}\right]} \delta y_{j} \\
& =\delta \sum_{j \in\left[1, i^{\prime}\right]} y_{j}+\frac{i^{\prime}}{n}(1-\delta) .
\end{aligned}
$$

By rearranging this equality we find that $\sum_{j \in\left[1, i^{\prime}\right]} y_{j}=i^{\prime} / n$. Since $y(N)=1$, we have that the average of $y_{1}, \ldots, y_{i^{\prime}}$ weakly exceeds the average of $y_{i^{\prime}+1}, \ldots, y_{n}$, a contradiction to Theorem 8.1.

Q.E.D.

We show next that procedural fairness results in the egalitarian solution.

THEOREM 8.4: The procedurally fair after-tax income is equal to $(1 / n)$ e.

Proof: Consider a sequence of continuation probabilities $\left(\delta_{m}\right)_{m \in \mathbb{N}}$ converging to 1 from below. Let $p_{m}$ be the tax equilibrium corresponding to continuation probability $\delta_{m}$. In view of Theorem 7.1 we can assume that the sequences $\left(p_{m}^{i}\right)_{m \in \mathbb{N}}$ converge to the same limit $\bar{p}$ for each $i \in N$. By Theorem 8.3 it holds that $p_{m, i}^{i}=p_{m, i+1}^{i}$ for every $m$ and every $i \in[1, n-1]$, so $\bar{p}_{i}=\bar{p}_{i+1}$ for every $i \in[1, n-1]$. We can conclude that $\bar{p}_{1}=\cdots=\bar{p}_{n}$, and $\bar{p}=(1 / n) e$.

Q.E.D.

\section{Conclusion}

We have addressed the consequences of procedurally fair income taxation in a very stylized model where citizens are risk-neutral and have no disutility from labor. Incentive compatibility dictates that after-tax income should be increasing in pre-tax income. There are no distortive effects from income taxation in our setting. These simplifying assumptions enable us to derive the stark result that procedural fairness results in complete redistribution.

These findings are in considerable contrast to those of Herings and Predtetchinski (2011b), where the same model was analyzed under the restriction that taxation schemes be linear. In that case the procedurally fair tax rate has been shown to be equal to the percentage of citizens with below average income. Apparently, when policy makers have the possibility to commit to linear taxation schemes, one would expect lower tax rates. Without such commitment possibilities, below average income citizens can make use of the flexibility of taxation schemes to increase the amount of redistribution in society.

The full flexibility of tax schedules studied here, up to the restrictions imposed by incentive compatibility, allows for all kinds of taxation schemes, including progressive ones. 
The high-dimensionality of the resulting policy space has hindered analysis in the literature, as Nash equilibria of majority voting games typically fail to exist. Roemer (1999) bypasses this problem by weakening the Nash equilibrium concept. Here, it is the requirement of unanimous agreement that enables us to deal with higher-dimensional policy spaces without having to resort to mixed-strategy Nash equilibria. In the limit equilibrium, however, no use of progressive taxation is made, but a linear taxation scheme involving full redistribution results.

At least two of the simplifying assumptions we have made, call for a generalization. On the one hand one would like to introduce risk-averse citizens, as risk aversion has been recognized as an important driving force for outcomes of bargaining models. For two-player bargaining models, it has been observed by Roth (1985) that increases in riskaversion undermine a player's bargaining position. If we assume decreasing absolute risk aversion, then the introduction of risk aversion should be expected to benefit high income citizens, leading to less than full redistribution. Secondly, one would like to see the effect of introducing disutility of labor. In this case, the usual incentive effects come into play, make full redistribution undesirable for all citizens.

\section{References}

Banks, J., And J. Duggan (2000), "A Bargaining Model of Collective Choice," American Political Science Review, 94, 73-88.

Binmore, K. (1987), "Perfect Equilibria in Bargaining Models," in K. Binmore and P. Dasgupta (eds.), The Economics of Bargaining, Basil Blackwell, Oxford, UK, pp. 77-105.

Bolton, G.E., J. Brandts, And A. Ockenfels (2005), "Fair Procedures: Evidence from Games Involving Lotteries," The Economic Journal, 115, 1054-1076.

Britz, V., P.J.J. Herings, And A. Predtetchinski (2010), "Non-cooperative Support for the Asymmetric Nash Bargaining Solution," Journal of Economic Theory, 145, 1951-1967.

Cardona, D., and C. Ponsatí (2007), "Bargaining One-dimensional Social Choices," Journal of Economic Theory, 1-25 pages.

Cho, S.J., And J. Duggan (2003), "Uniqueness of Stationary Equilibria in a One-dimensional Model of Bargaining," Journal of Economic Theory, 113, 118-130.

Fleurbaey, M., And F. Maniquet (2006), "Fair Income Tax," Review of Economic Studies, 73, 5583.

Hart, S., And A. Mas-Colell (1996), "Bargaining and Value," Econometrica, 64, 357-380.

Herings, P.J.J., And A. Predtetchinski (2011A), "On the Asymptotic Uniqueness of Bargaining Equilibria," Economics Letters, 111, 243-246.

Herings, P.J.J., And A. Predtetchinski (2011B), "Procedurally Fair Taxation," METEOR Research Memorandum 11/24, Maastricht University, Maastricht, pp. 1-25. 
Imai, H., And H. Salonen (2000), "The Representative Nash Solution for Two-sided Bargaining Problems," Mathematical Social Sciences, 39, 349-365.

Kalandrakis, T. (2006), "Regularity of Pure Strategy Equilibrium Points in a Class of Bargaining Games," Economic Theory, 28, 309-329.

Kultti, K., And H. Vartiainen (2010), "Multilateral Non-Cooperative Bargaining in a General Utility Space," International Journal of Game Theory, 39, 677-689.

Laruelle, A., And F. Valenciano (2007), "Bargaining in Committees as an Extension of Nash's Bargaining Theory," Journal of Economic Theory, 132, 291-305.

Merlo, A., And C. Wilson (1995), "A Stochastic Model of Sequential Bargaining with Complete Information," Econometrica, 63, 371-399.

Mirrlees, J.A. (1971), "An Exploration in the Theory of Optimum Income Taxation," Review of Economic Studies, 38, 175-208.

Miyakawa, T. (2008), "Noncooperative Foundation of $n$-Person Asymmetric Nash Bargaining Solution," Journal of Economics of Kwansei Gakuin University, 62, 1-18.

Roemer, J.E. (1999), "The Democratic Political Economy of Progressive Income Taxation," Econometrica, 67, 1-19.

Roth, A.E. (1985), "A Note on Risk Aversion in a Perfect Equilibrium Model of Bargaining," Econometrica, 53, 207-211.

Rubinstein, A. (1982), "Perfect Equilibrium in a Bargaining Model," Econometrica, 50, 97-109.

Thomson, W. (2003), "Axiomatic and Game-theoretic Analysis of Bankruptcy and Taxation Problems: A Survey," Mathematical Social Sciences, 45, 249-297. 